\title{
Paclitaxel - A Tool to Prevent Stenosis in Vascular Access for Haemodialysis?
}

\author{
Klaus Konner \\ Bergisch Gladbach, Germany
}

A well-functioning vascular access - an arteriovenous fistula (AVF) or an arteriovenous graft (AVG) - is the lifeline for the hemodialysis patient. Access dysfunction is mainly caused by venous stenosis which finally results in access thrombosis.

In forearm AVF, postanastomotic stenoses are observed in about $50 \%$ of patients; the underlying mechanism still remains speculative. In addition, AVF stenoses occur in scarred and narrowed venous segments due to cannulations during the predialysis period. Repeated cannulation of the identical area for dialysis access can lead to stenoses and aneurysms.

In $\mathrm{AVG}$, stenoses are predominantly observed at the graft-vein anastomosis caused by venous neointimal hyperplasia (VNH) which consists of smooth muscle cells, fibroblasts and myofibroblasts and a microvessel formation within the neointima causing a substantial economic burden and clinical morbidity. Numerous surgical and interventional therapeutical techniques were introduced. It therefore makes sense to look for an effective prevention of VNH.

Dr. Melhem and co-workers from Cincinnati, USA, report on 'Development of a local perivascular paclitaxel delivery system for hemodialysis vascular access dysfunction: Polymer preparation and in vitro activity'. They succeeded in developing a paclitaxel-loaded ethylene vinyl acetate (EVA) polymer wrap for local drug delivery in a perivascular configuration in a pig AVG model.
Paclitaxel has an antiproliferative efficacy against a variety of malignant cell lines and against smooth muscle cells [1, 2]. Hong et al. [3] reported on the placement of paclitaxel-eluting stents to reduce coronary in-stent restenosis in pigs. As an alternative to this 'inside-out' drugeluting stent approach, Melhem et al. present an 'outsidein' perivascular approach. They could demonstrate 'that the paclitaxel polymer wraps were mechanically stable with a burst release phase followed by a slower continuous release phase'. In addition, they showed that the 'released paclitaxel retains its physicochemical and biological properties and is able to inhibit the proliferation of smooth muscle cells, endothelial cells and fibroblasts in vitro'.

It seems extremely unlikely that the local delivery of paclitaxel would result in any systemic toxicity. A still critical issue in any delivery system, although a fascinating aspect, is the "need to match the temporal profile of drug release to the biologic sequence of events that characterize the disease process'.

Finally, the authors speculate that 'a potent antiproliferative drug milieu at the site of vascular injury for this prolonged period of time could result in a permanent alteration of cell types at the graft-vein or the artery-vein anastomosis, such that neointimal hyperplasia may not occur even in the absence of paclitaxel release after the complete release of paclitaxel from the EVA polymer wrap'.

\section{KARGER}

Fax +4161306 1234 E-Mail karger@karger.ch www.karger.com
(C) 2006 S. Karger AG, Basel 0253-5068/06/0243-0287\$23.50/0

www.karger.com/bpu
Klaus Konner, MD

Schau ins Land 24

DE-51429 Bergisch Gladbach (Germany)

Tel. +49 2204 56625, Fax +49 2204609691

E-Mail klaus.konner@uni-koeln.de 
First encouraging results with an in-vivo AVG model in pigs showed a complete absence of luminal stenosis at the wrapped graft-vein anastomosis compared with controls [4].

\section{Comment}

The idea to place a paclitaxel-loaded EVA polymer wrap in a perivascular position is original and raises, as all promising research issues do, a couple of questions.

(1) The impact of paclitaxel delivery on the process of graft endothelialization (not VNH!) should be undergoing further in-vivo research. Do additional mechanisms beyond the paclitaxel-bound effect play a substantial role?

(2) The transfer from pig to human conditions is still an open question.

(3) The technique to create the anastomoses in the pig model should be standardized as far as it is feasible: diameter of the feeding artery and draining vein and the diameter of the prosthetic graft, the angle between graft and vein, length of the graft-vein anastomosis, measurement of blood flow volume at standardized location and days after implantation.

(4) There may be different types/locations of use of the wrap paclitaxel delivery system technique in AVF and AVG. Preferred position of the paclitaxel wrap in AVF will be the postanastomotic venous segment. Preexisting venous narrowings would probably not respond to paclitaxel treatment. Other types of venous stenoses depend on numerous conditions caused by flow and/or cannulation characteristics and cannot be calculated in advance to place the perivascular wrap in an optimal location. In addition, insertion of the paclitaxel-loaded wrap would require a separate surgical exposure. The graft-vein anastomosis in AVG will be the by far preferred location for this paclitaxel delivery technique. Clinical experience suggests to cover the terminal $3 \mathrm{~cm}$ of the graft and the beginning $3 \mathrm{~cm}$ of the autologous vein, that means the region of the graft-vein anastomosis and the first venous segment.

(5) It would be fascinating to find an answer whether an initially performed successful paclitaxel delivery with an expected reduction/prevention of neointimal hyperplasia will be able to prevent late occurrence of graft-vein stenoses, e.g. after the first or second year of hemodialysis therapy.

(6) Furthermore, the wrap technique for delivery of paclitaxel may be measured against other modes of application, for example direct coating/impregnation of grafts.

(7) Finally, we should be aware that the application of paclitaxel represents only one tool in preventing postanastomotic stenoses in AVF and graft-vein stenoses in AVG. We should not underestimate the preexisting quality of the vessels involved - maybe that this is more important in human than in pig models. Additional factors are the surgical expertise, the design of the anastomosis (angle between graft and vein, length of anastomosis, use of 'hooded' PTFE grafts), interindividually different circulatory and rheological conditions. Surgical errors remain surgical errors, even with paclitaxel delivery.

Regardless of the complexity of these different aspects, the further steps in evaluating the local paclitaxel delivery should be done in pig models followed by welcomed research in a clinical setting. Hopefully, the paclitaxel delivery technique can be established as a tool to reduce substantially the troublesome, risky and costly rate of stenoses in vascular access for hemodialysis.

\section{References}

-1 Czuba ZP, Krol W, Hasinski P, Nowowiejska A: The effects of taxol (paclitaxel) on chemiluminescence of neutrophils, macrophages and J.774. 2 cell line. Acta Biochem Pol 1998;45: 103-106.

$\checkmark 2$ Heller PF: Paclitaxel and arterial smooth muscle cell proliferation. Circulation 1998;97: 1651 .
Hong MK, Kornowski R, Bramwell O, Ragheb AO, Leon MB: Paclitaxel-coated GianturcoRoubin II (GRII) stents reduce neointimal hyperplasia in a porcine coronary in-stent restenosis model. Coron Artery Dis 2001;12: 513-515.
4 Kelly B, Melhem M, Desai P, Kasting J, Zhang J, Li J, Heffelfinger S, Rudich S, Roy-Chaudhury P: Perivascular paclitaxel polymers reduce neointimal hyperplasia and venous stenosis in PTFE arteriovenous grafts: a solution for hemodialysis vascular access dysfunction? (Abstract) J Am Soc Nephrol 2004;15:25A26A. 